\title{
Shifting from normal to abnormal: Transformation of clinical presentation of post-laparoscopic Roux-en-Y gastric bypass surgery complications
}

\author{
David Widjaja MD, Bhavna Balar MD
}

B ariatric surgery can lead to considerable weight loss in patients with morbid obesity who have failed to achieve or maintain weight loss with supervised programs. Laparoscopic Roux-en-Y gastric bypass (LRYGB) and laparoscopic adjustable gastric banding are common bariatric surgeries. The former results in better weight loss and a reduced number of failures compared with the latter, despite its significantly longer operative time and life-threatening complications (1). We present a case involving a 32-year-old woman with history of LRYGB who presented with intractable abdominal pain. The initial abdominal imaging study was unremarkable; however, subsequent imaging studies showed volvulus of the midgut requiring surgical intervention.

\section{CASE PRESENTATION}

A 32-year-old woman with history of LRYGB surgery performed four years previously presented to Bronx Lebanon Hospital Center (New York, USA) because of worsening abdominal pain for three days. Since the surgery, she had developed intermittent epigastric pain, which was usually relieved with ranitidine or omeprazole. One day before presentation, she was evaluated in another hospital's emergency room. A computed tomography (CT) scan of the abdomen with oral and intravenous contrast showed nondilated gastric pouch and unremarkable gastrojejunal and jejunojejunal anastomoses. Because

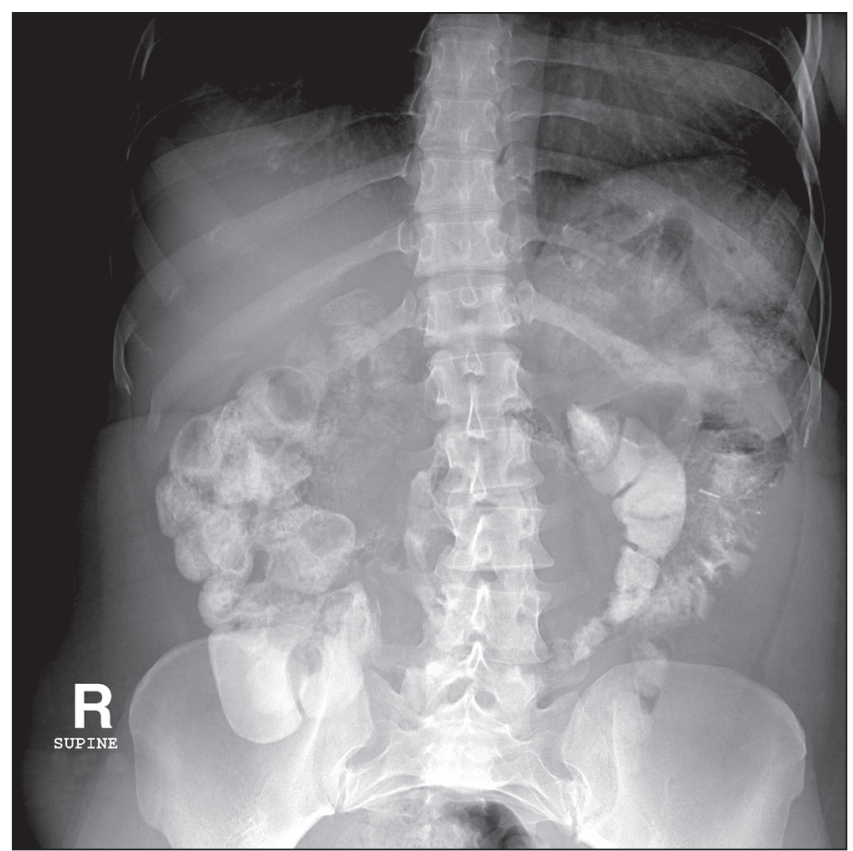

Figure 1) Initial of three views of abdominal $x$-ray showing no evidence of intestinal obstruction there were no significant radiological findings, she was discharged home. The pain persisted and worsened; therefore, she presented to Bronx Lebanon Hospital Center. The abdominal pain was diffuse and associated with severe back pain, nausea and vomiting. She preferred the knee-to-chest position because this posture relieved the abdominal and back pain. She did not have any other known chronic comorbid illnesses and never used nonsteroidal anti-inflammatory drugs. There was no history of illicit substance use, tobacco smoking or heavy consumption of alcohol. Laboratory tests revealed unremarkable serum electrolyte and liver enzyme levels, a normal serum lipase level and a serum lactic acid concentration of $2.3 \mathrm{mmol} / \mathrm{L}$. Three views of abdominal $\mathrm{x}$-ray in the emergency room performed $6 \mathrm{~h}$ after the previous abdominal CT scan revealed no evidence of intestinal obstruction (Figure 1). Oral contrast media from a previous abdominal CT scan was still visualized within the large bowel on the abdominal $\mathrm{x}$-ray. A repeat abdominal CT scan was delayed due to the unremarkable finding of the two previous imaging studies. Esophagogastroduodenoscopy showed a normal esophagus, patent gastric bypass, small volume of gastric pouch, healthy appearing mucosa of gastrojejunal and jejunojejunal anastomoses, and a short afferent limb. No lesions were identified during esophagogastroduodenoscopy to explain the abdominal pain. An abdominal sonogram performed for persistent abdominal pain showed a common bile duct diameter of $3 \mathrm{~mm}$, patent portal veins with normal hepatopetal direction of flow, and unremarkable visualized portions of the aorta and inferior vena cava. At this point, $32 \mathrm{~h}$ after the CT scan at another institution, a repeat CT scan of abdomen with oral and intravenous contrast was performed, which revealed a counter-clockwise swirling configuration of the superior mesenteric artery, superior mesenteric vein and mesentery (Figure 2). A similar swirl pattern of vasculature was apparent in the left upper quadrant area (Figure 2). The CT scan study also showed levoscoliosis of the lumbar spine, normal calibre

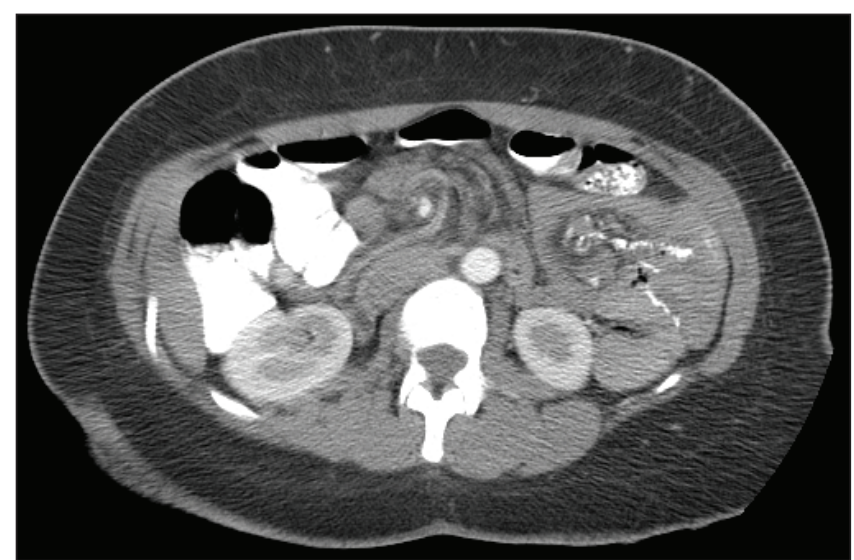

Figure 2) Computed tomography scan of the abdomen revealing a counterclockwise swirling configuration of vasculatures and mesentery

Division of Gastroenterology, Department of Medicine, Bronx Lebanon Hospital Center, Bronx, New York, USA

Correspondence: Dr David Widjaja, Division of Gastroenterology, Bronx Lebanon Hospital Center, 1650 Grand Concourse, Bronx, New York 10457,

USA. Telephone 718-518-5550, fax 718-518-5111, e-mail medicine.nyc@gmail.com

Received for publication August 9, 2012. Accepted August 18, 2012 


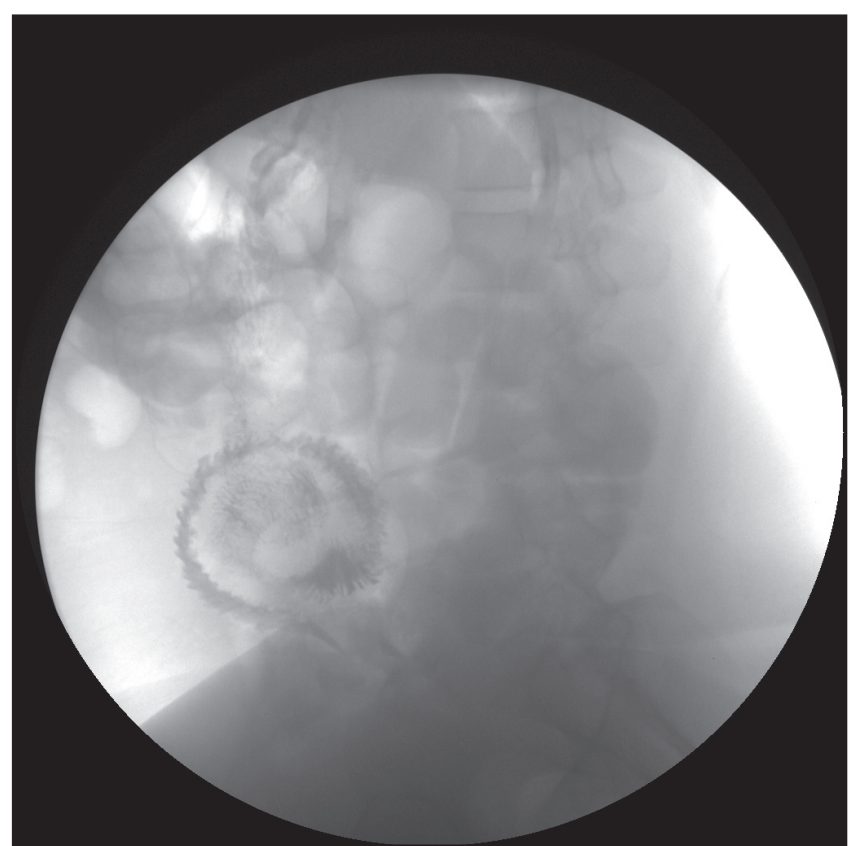

Figure 3) X-ray of a small bowel series showing swirling of the jejunal loops with thickening mucosa

of small bowels, no wall thickening or pneumatosis intestinalis, no evidence of bowel obstruction and progression of oral contrast to the colon. X-rays of a small bowel series, which was performed $78 \mathrm{~h}$ after the second abdominal CT scan, revealed swirling of the jejunal loops with thickening mucosa, which raised suspicion of a midgut volvulus (Figure 3). Diagnostic laparoscopy was performed due to the clinical and radiological findings. Intra-abdominal inspection during the laparoscopic procedure revealed a markedly dilated transverse colon and small bowel, with a dusky colour in several regions; however, it appeared viable. Several adhesions were indentified from the omentum down to the pelvis, which were divided using cautery. One loop of bowel was untwisted after the adhesion was divided. The laparoscopic procedure was then converted to open abdominal exploration because the surgeon was unable to follow the bowel from the ileocecal valve back to the jejunojenostomy without encountering a twisted mesenteric area. Open abdominal exploration showed that the bowel was twisted in multiple locations and the entire bowel had herniated through an internal hernia. The entire bowel was then untwisted and laid out in its proper orientation without any twist in the mesentery. The bowel became pink and was viable for its entire length. A mesenteric defect was found at the jejunojejunostomy site. The defect was closed using a 3-0 silk running suture. Also present was an antecolic Roux limb with a wide-open space underneath it. The transverse colon was found on the right side of the Roux limb, which appeared to be the cause of the colonic distention. The transverse colon and its mesentery were then moved to the left side of the Roux limb. Following surgery, she was stable and able to tolerate oral intake. Repeat x-ray of small bowel showed resolution of the previous internal hernia (Figure 4). Oral contrast media was seen passing into the jejunum and there was no evidence of contrast extravasations on this particular imaging study. The patient was discharged home without significant abdominal symptoms on postoperative day 4 .

\section{DISCUSSION}

Late complications of LRYGB include anastomotic stricture, cholelithiasis, marginal ulceration, internal hernia and intestinal obstruction $(2,3)$. Anastomotic stricture is usually associated with nausea, vomiting, dysphagia and intolerance to oral intake. The clinical presentations of the other complications usually include abdominal pain. In symptomatic cholelithiasis, patients typically experience biliary-type

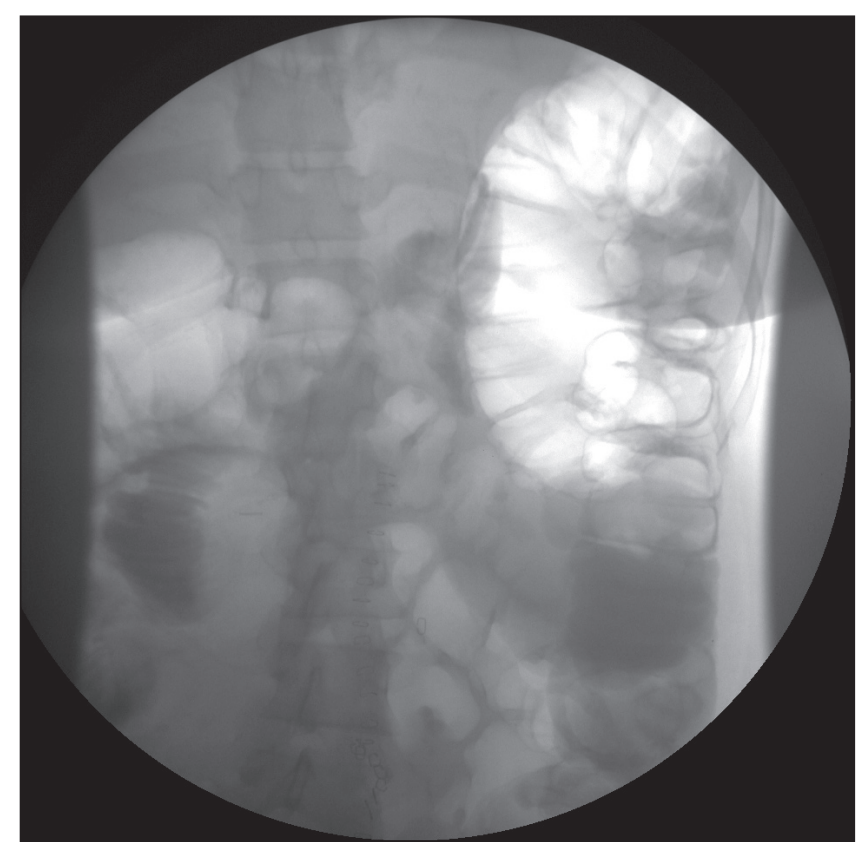

Figure 4) X-ray of small bowel showing resolution of previous internal hernia

pain, increased liver enzyme levels, dilated common bile duct or jaundice. Patients with marginal ulceration usually present with nausea, bleeding, perforation or fistulization. Endoscopic study confirms the presence of marginal ulcer(s). Most cases of marginal ulceration respond to acid-reducing agents such as proton pump inhibitors or histamine-2 receptor antagonists (4). Therefore, a marginal ulcer was unlikely to be the cause of our patient's intractable symptoms.

Symptoms of internal hernia consist of abdominal pain, nausea and vomiting, which may be acute and severe, intermittent or very vague. In the presence of intermittent or vague symptoms, routine laboratory examinations may not be helpful because most blood parameters will be within the normal range (5). Upper gastrointestinal imaging studies often lead to the diagnosis of internal hernia (6). Abdominal CT scan study of our patient showed a mesenteric swirl, which is considered to be the best indicator on CT scan of an internal hernia following LRYGB surgery $(7,8)$. However, negative findings on radiological investigations should not be used to rule out the diagnosis of internal hernia because interpretation of radiological findings in a patient who has undergone LRYGB surgery may be difficult in the absence of specific knowledge of the bariatric surgery (9). Furthermore, the occurrence of abdominal, colicky pain after LRYGB surgery, even in the absence of other symptoms, altered laboratory and/or radiological findings, should raise the suspicion of internal hernia, and surgical exploration should be considered if pain persists or recurs (10). Our patient may have had a minimal internal hernia without bowel obstruction during the initial presentation; therefore, the first abdominal CT scan was read as unremarkable by the radiologist. Once the entire small bowel had migrated through the mesentery and rotated on its vascular supply, the symptoms became more severe and, finally, obvious signs of volvulus and internal hernia emerged from the subsequent imaging studies.

There are three sites where an internal hernia after LRYGB may occur: through the transverse mesocolon defect; through the enteroenterostomy mesenteric defect; and through the space between mesentery of Roux limb and transverse colon (ie, Petersen's space) (5). Overall, the site of internal hernia varies with transverse mesocolon hernias being the most common, followed by enteroenterostomy and then Peterson's space hernia. However, in antecolic LRYGB, as in our patient, internal hernias are equally distributed between Peterson's space and the enteroenterostomy defect (11). 
Our patient's symptoms of back pain and relieving knee-to-chest postures are not typical of internal hernia and have not been reported to date. The midgut volvulus may have magnified the symptoms of her scoliosis. The present report emphasizes the importance of suspecting internal herniation in patients with history of LRYGB surgery. A repeat abdominal imaging study should be strongly considered if symptoms become intractable.

\section{REFERENCES}

1. Angrisani L, Lorenzo M, Borrelli V. Laparoscopic adjustable gastric banding versus Roux-en-Y gastric bypass: 5 -year results of a prospective randomized trial. Surg Obes Relat Dis 2007;3:127-32.

2. Shiffman ML, Sugerman HJ, Kellum JM, Brewer WH, Moore EW. Gallstone formation after rapid weight loss: A prospective study in patients undergoing gastric bypass surgery for treatment of morbid obesity. Am J Gastroenterol 1991;86:1000-5.

3. Al Harakeh AB. Complications of laparoscopic Roux-en-Y gastric bypass. Surg Clin North Am 2011;91:1225-37.

4. Sugerman HJ. Gastric bypass surgery for severe obesity. Semin Laparosc Surg 2002;9:79-85.

5. Iannelli A, Facchiano E, Gugenheim J. Internal hernia after laparoscopic Roux-en-Y gastric bypass for morbid obesity. Obes Surg 2006;16:1265-71.
6. Blachar A, Federle MP. Gastrointestinal complications of laparoscopic roux-en-Y gastric bypass surgery in patients who are morbidly obese: Findings on radiography and CT. Am J Roentgenol 2002;179:1437-42.

7. Iannuccilli JD, Grand D, Murphy BL, Evangelista P, Roye GD, Mayo-Smith W. Sensitivity and specificity of eight CT signs in the preoperative diagnosis of internal mesenteric hernia following Roux-en-Y gastric bypass surgery. Clin Radiol 2009;64:373-80.

8. Lockhart ME, Tessler FN, Canon CL, et al. Internal hernia after gastric bypass: Sensitivity and specificity of seven CT signs with surgical correlation and controls. AJR Am J Roentgenol 2007;188:745-50.

9. Onopchenko A. Radiological diagnosis of internal hernia after Roux-en-Y gastric bypass. Obes Surg 2005;15:606-11.

10. Champion JK, Williams M. Small bowel obstruction and internal hernias after laparoscopic Roux-en-Y gastric bypass. Obes Surg 2003;13:596-600.

11. Ahmed AR, Rickards G, Husain S, Johnson J, Boss T, O'Malley W. Trends in internal hernia incidence after laparoscopic Roux-en-Y gastric bypass. Obes Surg 2007;17:1563-6.

The Canadian Journal of Gastroenterology is considering a limited number of submissions for IMAGE OF THE MONTH. These are based on endoscopic, histological, radiological and/or patient images, which must be anonymous with no identifying features visible. The patient must consent to publication and the consent must be submitted with the manuscript. All manuscripts should be practical and relevant to clinical practice, and not simply a case report of an esoteric condition. The text should be brief, structured as CASE PRESENTATION and DISCUSSION, and not more than 700 words in length. A maximum of three images can be submitted and the number of references should not exceed five. The submission may be edited by our editorial team. 


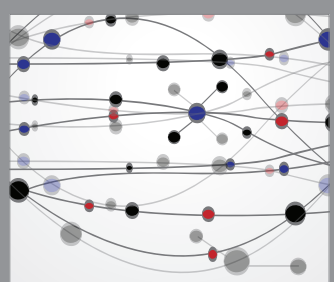

The Scientific World Journal
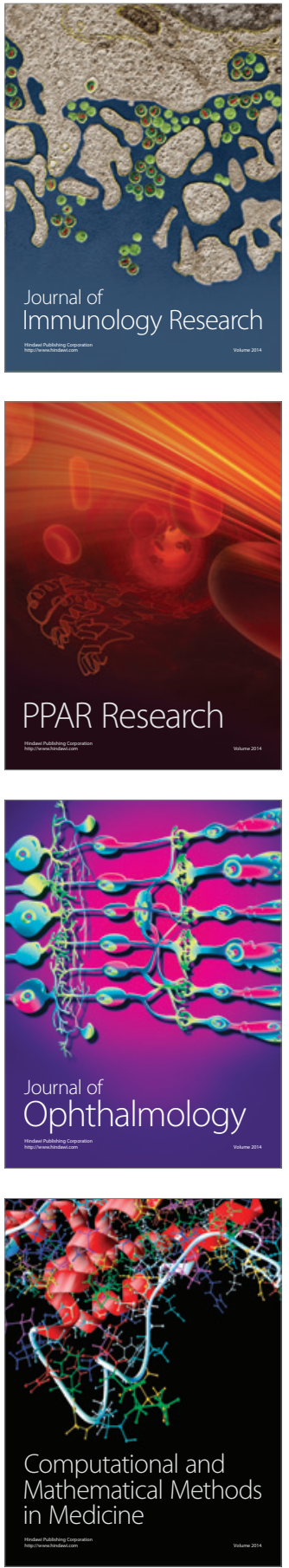

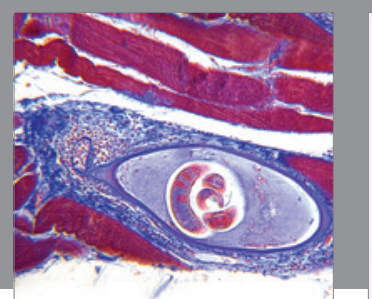

Gastroenterology Research and Practice

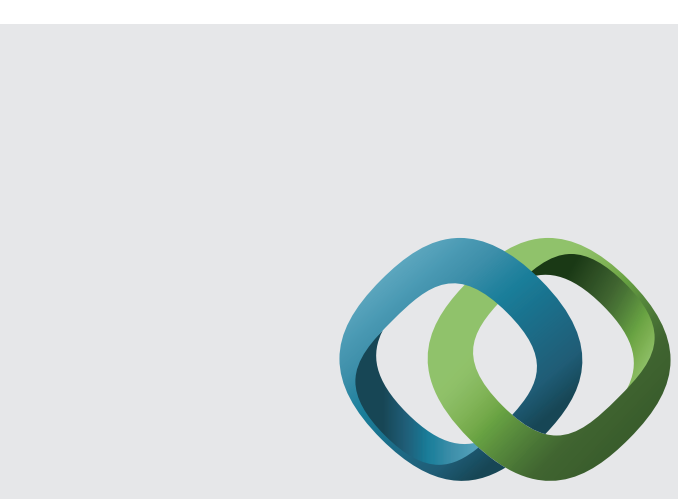

\section{Hindawi}

Submit your manuscripts at

http://www.hindawi.com
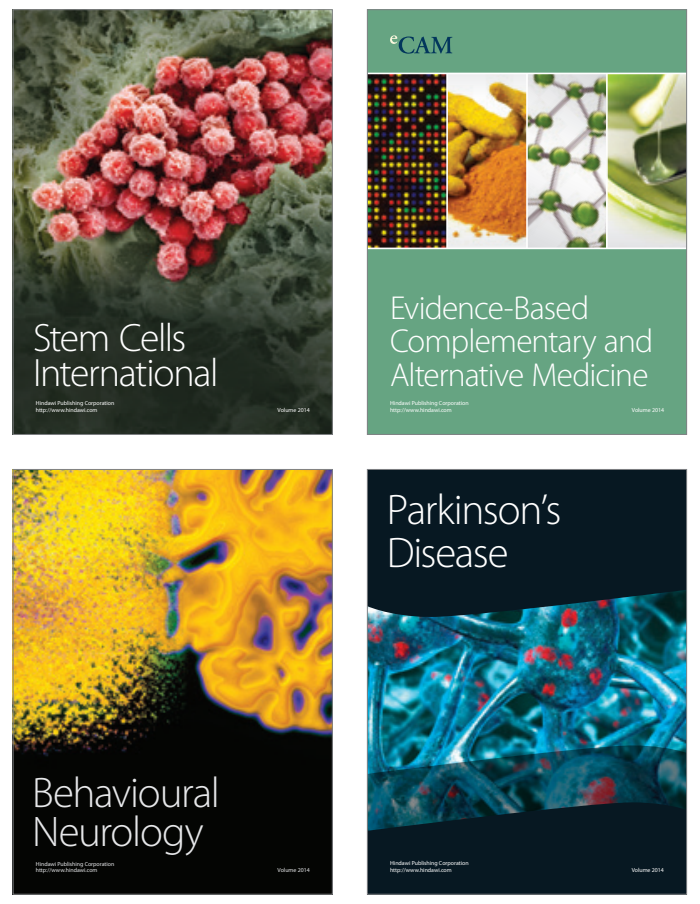
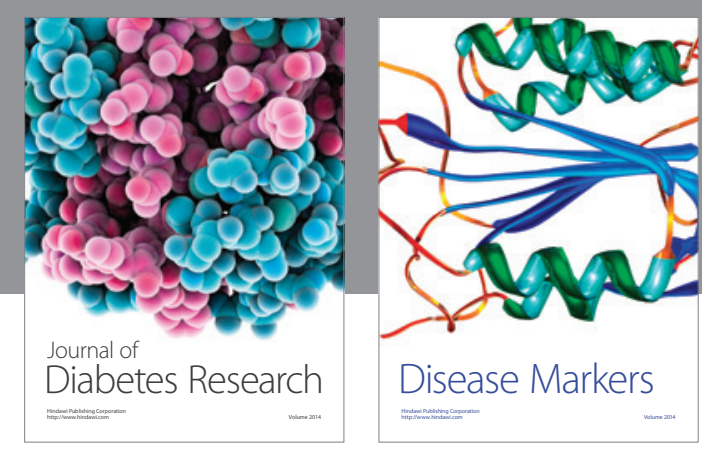

Disease Markers
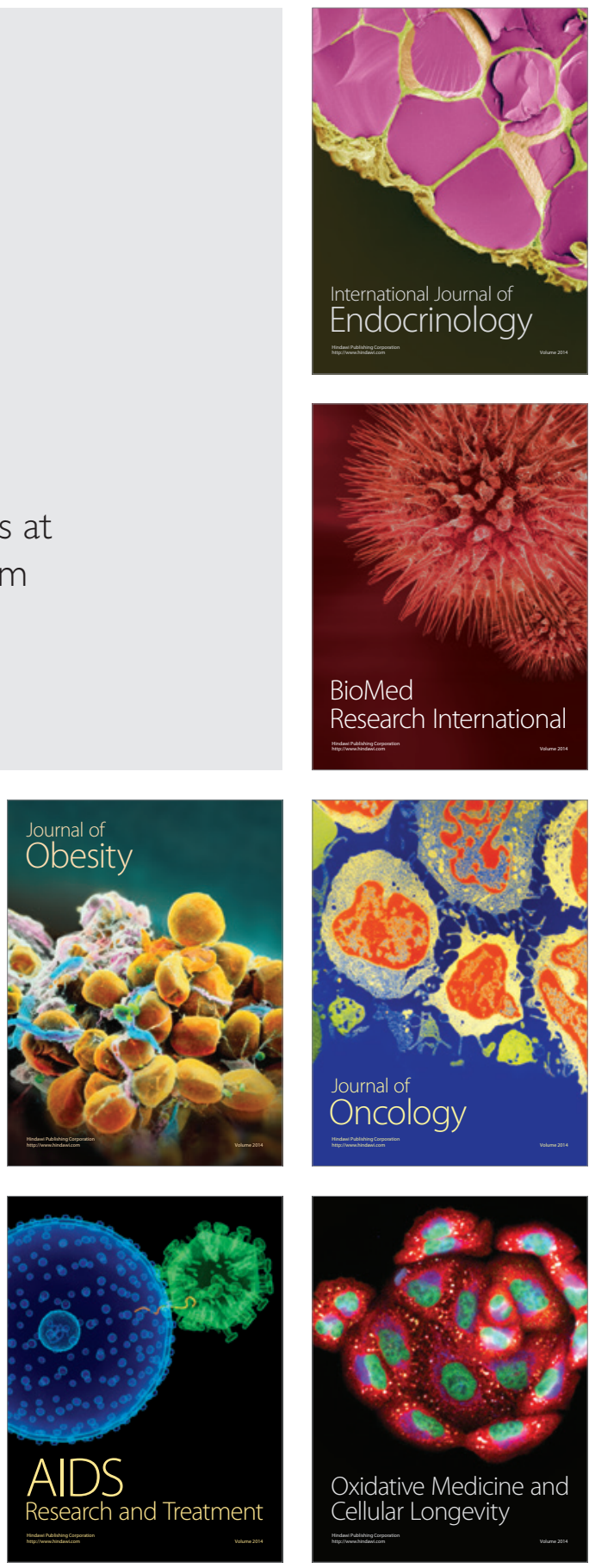